International Journal of Pure and Applied Mathematics

Volume 83 No. 1 2013, 81-90

ISSN: 1311-8080 (printed version); ISSN: 1314-3395 (on-line version)

url: http://www.ijpam.eu

doi: http://dx.doi.org/10.12732/ijpam.v83i1.7

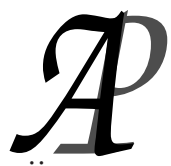

ijpam.eu

\title{
A NOTE ON HIGHER-ORDER CONVEXITY OF SEQUENCES
}

\author{
Katarína Nicová \\ Institute of Mathematics \\ Faculty of Science \\ Pavol Jozef Šafárik University in Košice \\ Jesenná 5, SK 04001 Košice, SLOVAKIA
}

\begin{abstract}
We present two different kinds of higher-order convexity of real sequences and investigate their basic properties. We also discuss their relationship with the usual higher-order convexity defined using the difference operators.
\end{abstract}

AMS Subject Classification: 26A51

Key Words: higher-order convexity, real sequence, monotonicity, inequality

\section{Introduction}

Let $S$ be a set of all real sequences. A sequence $\left(a_{n}\right)_{1}^{\infty} \in S$ is called convex if $a_{n+2}-2 a_{n+1}+a_{n} \geq 0$ for all $n \in \mathbb{N}$. For some properties of convex sequences and their applications see e.g. [2], [11] [12], [13] and [15]. Various generalizations of convexity of sequences were studied by various authors. In [9] a sequence $\left(a_{n}\right)_{1}^{\infty} \in S$ is called $r$-convex for a positive real number $r$ if $L_{r} a_{n} \geq 0$ for all $n \in \mathbb{N}$, where the operator $L_{r}$ is given by $L_{r} a_{n}=a_{n+2}-(1+r) a_{n+1}+r a_{n}$. A similar generalization uses the operator $\Delta_{p, q} a_{n}=a_{n+2}+(p+q) a_{n+1}+p q a_{n}$ with $p, q \in \mathbb{R}$, see [8].

Received: September 25, 2012

(c) 2013 Academic Publications, Ltd. url: www.acadpubl.eu 
On the other hand the usual definition of convexity of higher order was introduced using the difference operators in [14] as follows. For a given sequence $\left(a_{n}\right)_{1}^{\infty} \in S$ let us define the difference operator of order $k$, or $k$-th difference operator, $k \in \mathbb{N}_{0}$, as

$$
\Delta^{0} a_{n}=a_{n}, \quad \Delta^{1} a_{n}=a_{n+1}-a_{n}, \quad \Delta^{k+1} a_{n}=\Delta^{1}\left(\Delta^{k} a_{n}\right) .
$$

In the case $k=1$ we usually write $\Delta a_{n}$ instead of $\Delta^{1} a_{n}$. It is also possible to define difference operator for $k \in \mathbb{R}$, see [10]. Using this notion we may define the generalized convexity of sequence.

Definition 1.1. Let $k \in \mathbb{N}_{0}$. A sequence $\left(a_{n}\right)_{1}^{\infty}$ is called $(\Delta, k)$-convex, if $\Delta^{k} a_{n} \geq 0$ for all $n \in \mathbb{N}$.

We prefer the notation $(\Delta, k)$-convex to emphasize the fact that it is defined using the difference operator and also from the fact that the notation $k$-convex is reserved for convexity which we introduce in the next section. Clearly, every non-negative sequence is $(\Delta, 0)$-convex, every non-decreasing sequence is $(\Delta, 1)$-convex and $(\Delta, 2)$-convex sequence is equivalent to the standard convex sequence.

The set $S$ of all sequences becomes a vector space if it is supplied by addition and multiplication by scalars in the usual way. Let us denote by $K_{k}^{\Delta} \subset S$ the set of all $(\Delta, k)$-convex sequences. From the linearity of $k$-th difference operator it follows that $K_{k}^{\Delta}$ is closed under the addition and non-negative scalar multiplication of sequences. Let us mention that $(\Delta, k)$-convexity of sequence is used in solving Hausdorff moment problem for operators [3], or in Cesàro summability of Fourier series [1].

We observe that in the definition of convexity the arithmetic mean is used. Using different kind of means we may obtain different kinds of convexity, for instance, geometric mean leads to the log-convexity. It is also possible to use other types of means, such as discrete version of generalized weighted means introduced and investigated in [4], [5], [6] and [7], in general.

In this paper we introduce two different types of higher-order convexity and present their basic properties. At the end we give some examples demonstrating relationships among these types of generalized convexity and $(\Delta, k)$-convexity. 


\section{2. $k$-Convexity and its Basic Properties}

Definition 2.1. Let $k$ be a fixed natural number, $k \geq 2$. A sequence $\left(a_{n}\right)_{1}^{\infty}$ is called $k$-convex, if for all $n \in \mathbb{N}$ holds

$$
a_{n+1} \leq a_{n}+\frac{a_{n+k}-a_{n}}{k}, \quad \text { and } \quad a_{n+k-1} \leq a_{n}+\frac{(k-1)\left(a_{n+k}-a_{n}\right)}{k} .
$$

For a $k$-convex sequence $\left(a_{n}\right)_{1}^{\infty}$ we may glue the two expressions in (1) to get the inequality

$$
a_{n+k}-a_{n+k-1} \geq a_{n+1}-a_{n} .
$$

This observation is the motivation for our study because the usual convexity (or, $(\Delta, 2)$-convexity) may be rewritten as $a_{n+2}-a_{n+1} \geq a_{n+1}-a_{n}$. Therefore we are interested in question: What if we replace the left-hand side of the above inequality by a more general term depending on natural $k \geq 2$ ?

Remark 2.2. Clearly, if a sequence $\left(a_{n}\right)_{1}^{\infty}$ is $k$-convex, then the inequality (2) holds, but not vice versa. For instance, it is easy to verify that the sequence $\left(a_{n}\right)_{1}^{\infty}=\left((-1)^{n}\right)_{1}^{\infty}$ is not $k$-convex for any $k$, whereas

$$
a_{n+k}-a_{n+k-1}-a_{n+1}+a_{n}=2(-1)^{n}\left[(-1)^{k}+1\right],
$$

and thus for each $k=2 m+1, m \in \mathbb{N}$, it fulfills the inequality (2), but for any $k=2 m, m \in \mathbb{N}$, it does not. Further examples may be found in Section 3 . We also mention that in the expression (2) only four summands appear unlike $(\Delta, k)$-convexity where we have $k+1$ summands. Therefore this new type of convexity given by (2) seems to be much easier to use for practical purposes, but we will not study it here in detail.

Furthermore, we may analogously introduce notions of strictly $k$-convex, $k$-concave and strictly $k$-concave sequence replacing signs $\geq$ in Definition 2.1 by $>$, $\leq$ and $<$, respectively. Let us denote by $K_{k} \subset S$ (resp. $K_{k}^{s} \subset S$ ) the set of all $k$-convex (resp. strictly $k$-convex) sequences. Obviously, 2-convex sequence is equivalent to the usual convex sequence. A few basic properties of $k$-convexity are summarized in the following theorem which is easy to prove.

Theorem 2.3. Let $\left(a_{n}\right)_{1}^{\infty} \in K_{k}$.

(i) If $c>0$ and $d \in \mathbb{R}$, then $\left(c a_{n}+d\right)_{1}^{\infty} \in K_{k}$.

(ii) If $c \in \mathbb{R}$, then $\left(a_{n}+c n\right)_{1}^{\infty} \in K_{k}$.

(iii) If $a_{n} \geq 0$ for all $n \in \mathbb{N}$, then $\left(a_{n}^{2}\right)_{1}^{\infty} \in K_{k}$. 
(iv) If $\left(b_{n}\right)_{1}^{\infty} \in K_{k}$, then $\left(a_{n}+b_{n}\right)_{1}^{\infty} \in K_{k}$.

On the strength of these results we have that $K_{k} \subset S$ is closed under the addition and (positive) scalar multiplication of sequences.

Theorem 2.4. Let $\left(a_{n}\right)_{1}^{\infty} \in K_{k}$.

(i) If $\left(a_{n}\right)_{1}^{\infty}$ is non-decreasing, then $\left(n a_{n}\right)_{1}^{\infty} \in K_{k}$.

(ii) If $\left(\frac{a_{n}}{n}\right)_{1}^{\infty}$ is non-increasing, then $\left(\frac{a_{n}}{n}\right)_{1}^{\infty} \in K_{k}$.

Proof. (i) Since $\left(a_{n}\right)_{1}^{\infty}$ is $k$-convex, then

$$
\begin{aligned}
k(n+1) a_{n+1} & \leq(k-1)(n+1) a_{n}+(n+1) a_{n+k} \\
& =(k-1) n a_{n}+(n+k) a_{n+k}+(k-1)\left(a_{n}-a_{n+k}\right)
\end{aligned}
$$

Since $\left(a_{n}\right)_{1}^{\infty}$ is non-decreasing, then $a_{n}-a_{n+k} \leq 0$, and therefore

$$
(n+1) a_{n+1} \leq n a_{n}+\frac{(n+k) a_{n+k}-n a_{n}}{k} .
$$

Similarly,

$$
\begin{aligned}
k(n+k-1) a_{n+k-1} & \leq(n+k-1) a_{n}+(k-1)(n+k-1) a_{n+k} \\
& =n a_{n}+(k-1)(n+k) a_{n+k}+(k-1)\left(a_{n}-a_{n+k}\right),
\end{aligned}
$$

and the same argument as above leads to the inequality

$$
(n+k-1) a_{n+k-1} \leq n a_{n}+\frac{(k-1)\left((n+k) a_{n+k}-n a_{n}\right)}{k},
$$

i.e., the sequence $\left(n a_{n}\right)_{1}^{\infty}$ is $k$-convex.

(ii) Suppose the contrary, i.e., let there exist $N \geq 1$ such that $k \frac{a_{N+1}}{N+1}>$ $\frac{(k-1)}{N} a_{N}+\frac{a_{N+k}}{N+k}$. Then

$$
\begin{aligned}
k a_{N+1} & >\frac{(k-1)(N+1)}{N} a_{N}+\frac{(N+1)}{N+k} a_{N+k} \\
& =(k-1) a_{N}+a_{N+k}+(k-1)\left(\frac{a_{N}}{N}-\frac{a_{N+k}}{N+k}\right) \\
& \geq(k-1) a_{N}+a_{N+k},
\end{aligned}
$$

i.e., a contradiction, where the latter inequality follows from the fact that $\left(\frac{a_{n}}{n}\right)_{1}^{\infty}$ is non-increasing. 
Theorem 2.5. Let $\left(a_{n}\right)_{1}^{\infty} \in K_{k}^{s}$. Then one of the following cases may occur:

a) sequence $\left(a_{n}\right)_{1}^{\infty}$ is decreasing;

b) sequence $\left(a_{n}\right)_{1}^{\infty}$ is increasing;

c) there exists $N \in \mathbb{N}$ such that the sequence $\left(a_{l}\right)_{1}^{N}$ is decreasing and the sequence $\left(a_{m}\right)_{N+k-1}^{\infty}$ is increasing.

Proof. If for all $n \geq 1$ holds $a_{n+1}-a_{n}<0$, then the case a) occurs. If there exists $n \in \mathbb{N}$ such that $a_{n+1}-a_{n}>0$, take the first such index and denote it by $N$.

1) Let $N=1$. Firstly, from inequalities $a_{1}<a_{2}, a_{2}<a_{1}+\frac{a_{k+1}-a_{1}}{k}$ follows $a_{1}<a_{k+1}$. Secondly,

$$
a_{k}<a_{1}+\frac{(k-1)\left(a_{k+1}-a_{1}\right)}{k}<a_{1}+a_{k+1}-a_{1}=a_{k+1} .
$$

We will show that for all $n \geq k$ the inequalities $a_{n+1-k}<a_{n+1}$ and $a_{n}<a_{n+1}$ follow. The case $n=k$ we have just proved. Let the inequality hold for $n>k$ and show that it hold for $n+1$. From Definition 2.1 we get

$$
a_{n+2-k}<a_{n+1-k}+\frac{a_{n+1}-a_{n+1-k}}{k}<a_{n+1-k}+a_{n+1}-a_{n+1-k}=a_{n+1},
$$

as well as

$$
a_{n+1}<a_{n+2-k}+\frac{(k-1)\left(a_{n+2}-a_{n+2-k}\right)}{k}
$$

which implies $a_{n+2-k}<a_{n+2}$. Also, on the other hand

$$
a_{n+1}<a_{n+2-k}+\frac{(k-1)\left(a_{n+2}-a_{n+2-k}\right)}{k}<a_{n+2-k}+a_{n+2}-a_{n+2-k}=a_{n+2},
$$

i.e., the inequalities hold for all $n \geq k$, and therefore the case b) occurs.

2) Let $N>1$. Then for the sequence $a_{N}, a_{N+1}, \ldots$, we use assertion from 1 ) and obviously the case c) occurs.

Corollary 2.6. If $\left(a_{n}\right)_{1}^{\infty} \in K_{k}^{s}$ has some decreasing subsequence, then $\left(a_{n}\right)_{1}^{\infty}$ is decreasing.

Example 2.7. Let $\left(a_{n}\right)_{1}^{\infty}$ be a sequence given by $a_{2 n-1}=\log \frac{2 n+1}{2 n-1}$ and $a_{2 n}=\log \frac{n+1}{n}$. Then $\left(a_{n}\right)_{1}^{\infty} \in K_{2}^{s}$. Indeed,

$$
2 \log \frac{n+1}{n}<\log \frac{2 n+1}{2 n-1}+\log \frac{2 n+3}{2 n+1},
$$


which is equivalent to $\left(\frac{n+1}{n}\right)^{2}<\frac{2 n+3}{2 n-1}$, and this implies $\frac{1}{n^{2}(2 n-1)}>0$ for all $n \in \mathbb{N}$. The subsequence $\left(a_{2 n}\right)_{1}^{\infty}$ is decreasing because

$$
a_{2 n}>a_{2(n+1)} \Longleftrightarrow \log \frac{n+1}{n}>\log \frac{n+2}{n+1} \quad \text { for all } n \in \mathbb{N},
$$

which is equivalent to $\frac{1}{n(n+1)}>0$ for $n \in \mathbb{N}$. Then by Corollary 2.6 the sequence $\left(a_{n}\right)_{1}^{\infty}$ is decreasing.

Lemma 2.8. If $\left(a_{n}\right)_{1}^{\infty} \in K_{2}^{s}$ is not decreasing, then $\lim _{n \rightarrow \infty} a_{n}=+\infty$.

Proof. By Theorem 2.5 (for $k=2$ ) the sequence $\left(a_{n}\right)_{1}^{\infty}$ is increasing from an index $N \in \mathbb{N}$. Let us denote this sequence by $b_{1}, b_{2}, \ldots$ We claim that for arbitrary $n \geq 3$ and $1 \leq k \leq n-2$ holds

$$
b_{n}>(k+1) b_{n-k}-k b_{n-k-1} .
$$

For $k=1$ it reduces to $b_{n}>2 b_{n-1}-b_{n-2}$ which follows from strict convexity of $\left(a_{n}\right)_{1}^{\infty}$. Let us suppose that (3) is true for $k-1$. Then

$$
\begin{aligned}
b_{n} & >k b_{n-k+1}-(k-1) b_{n-k}>2 k b_{n-k}-k b_{n-k-1}-(k-1) b_{n-k} \\
& =(k+1) b_{n-k}-k b_{n-k-1} .
\end{aligned}
$$

Thus, for $k=n-2$ holds $b_{n}>(n-1) b_{2}-(n-2) b_{1}=(n-2)\left(b_{2}-b_{1}\right)+b_{2}$. Since $b_{2}-b_{1}>0$, then $\lim _{n \rightarrow \infty} b_{n}=\lim _{n \rightarrow \infty} a_{n}=+\infty$.

Now we are able to formulate analogous more general result including sequences from $K_{k}^{s}$.

Theorem 2.9. If $\left(a_{n}\right)_{1}^{\infty} \in K_{k}^{s}$ is not decreasing, then $\lim _{n \rightarrow \infty} a_{n}=+\infty$.

Proof. By Theorem 2.5 the sequence $\left(a_{n}\right)_{1}^{\infty}$ is increasing from an index $N$. Surely, the limit exists (at least improper one), and it is enough to investigate some its subsequence $\left(a_{r k}\right)_{r=1}^{\infty}$. From the assumption of strict $k$-convexity of sequence $\left(a_{n}\right)_{1}^{\infty}$ the inequalities

$$
\begin{aligned}
a_{(r+1) k} & <\frac{1}{k} a_{r k+1}+\frac{k-1}{k} a_{(r+1) k+1}, \\
a_{r k+1} & <\frac{k-1}{k} a_{r k}+\frac{1}{k} a_{(r+1) k}, \\
a_{(r+1) k+1} & <\frac{k-1}{k} a_{(r+1) k}+\frac{1}{k} a_{(r+2) k}
\end{aligned}
$$


follows for $r \geq 1$, thus we get the inequality

$$
a_{(r+1) k}<\frac{k-1}{k^{2}} a_{r k}+\frac{1}{k^{2}} a_{(r+1) k}+\frac{(k-1)^{2}}{k^{2}} a_{(r+1) k}+\frac{k-1}{k^{2}} a_{(r+2) k},
$$

which is equivalent to $\left(k^{2}-(k-1)^{2}-1\right) a_{(r+1) k}<(k-1) a_{r k}+(k-1) a_{(r+2) k}$. From it follows that $2 a_{(r+1) k}<a_{r k}+a_{(r+2) k}, r \in \mathbb{N}$, which means that the sequence $\left(a_{r k}\right)_{r=1}^{\infty}$ is strictly convex. By Lemma 2.8 we have $\lim _{r \rightarrow \infty} a_{r k}=\lim _{n \rightarrow \infty} a_{n}=+\infty$.

Example 2.10. Let us consider the sequence $\left(b_{n}\right)_{1}^{\infty}=\left(\frac{n !}{a^{n}}\right)_{1}^{\infty}$ for $a>0$. Then

$$
2 \frac{n !}{a^{n}}<\frac{(n-1) !}{a^{n-1}}+\frac{(n+1) !}{a^{n+1}}
$$

if and only if $2 a n<a^{2}+n^{2}+n$ for all $n \in \mathbb{N}$. It means that $\left(b_{n}\right)_{1}^{\infty} \in K_{2}^{s}$. Further, let us suppose that $\left(b_{n}\right)_{1}^{\infty}$ is decreasing, i.e., $\frac{(n-1) !}{a^{n-1}}>\frac{n !}{a^{n}}$ for all $n \in \mathbb{N}$. Then $a>n$ for all $n \in \mathbb{N}$ which contradicts the Archimedean property. Therefore, by Theorem 2.8 we get the well-known result $\lim _{n \rightarrow \infty} \frac{n !}{a^{n}}=+\infty$.

\section{A Few Examples}

In what follows we compare our new type of convexity with the usual $(\Delta, k)$ convexity. Obviously, $K_{2}^{\Delta}=K_{2}$ as already mentioned. On the strength of assertions we may say that for each $k \geq 2$ we have $K_{k} \cap K_{k}^{\Delta} \neq \emptyset$. However, for $k \geq 3$ we cannot expect that $K_{k} \subseteq K_{k}^{\Delta}$, or even $K_{k}^{\Delta} \subseteq K_{k}$. It is well-known that for an arbitrary $k \in \mathbb{N}_{0}$ and $n \in \mathbb{N}$ it holds

$$
\Delta^{k} a_{n}=\sum_{i=0}^{k}(-1)^{k+i}\left(\begin{array}{c}
k \\
i
\end{array}\right) a_{n+i} .
$$

Thus, (2) is not equal to (4) for each sequence. Of course, there exist sequences which are both $k$-convex and $(\Delta, k)$-convex (e.g. all constant sequences). Moreover, by the equality

$$
\sum_{i=0}^{k}(-1)^{k+i}\left(\begin{array}{l}
k \\
i
\end{array}\right)=0
$$

for arbitrary $k \in \mathbb{N}$ we have that the sequence $\left(a_{n}\right)_{1}^{\infty}=(c n+d)_{1}^{\infty}, c, d \geq$ 0 , is $(\Delta, k)$-convex for each $k \in \mathbb{N}_{0}$, as well as $k$-convex which follows from Theorem 2.3(ii). Immediately, an interesting question about structure of such sequence sets arises: how does the class of sequences both $k$-convex and $(\Delta, k)$ convex look? 
Example 3.1. The sequence $\left((-1)^{n}\right)_{1}^{\infty}$ is not $(\Delta, k)$-convex for any $k \in \mathbb{N}_{0}$ because

$$
\Delta^{k}(-1)^{n}=\sum_{i=0}^{k}(-1)^{k+n+2 i}\left(\begin{array}{l}
k \\
i
\end{array}\right)=(-1)^{k+n} \sum_{i=0}^{k}\left(\begin{array}{l}
k \\
i
\end{array}\right)=(-1)^{k+n} 2^{k} .
$$

As we have mentioned in Remark 2.2 this sequence is not $k$-convex for any $k \geq 2$, but for $k=2 m+1, m \in \mathbb{N}$, it fulfills the inequality (2).

Example 3.2. Let us consider the sequence $\left(a_{n}\right)_{1}^{\infty}=\left(\frac{1}{3}\left(3+6 n-2 n^{2}\right)\right)_{1}^{\infty}$. Then

$$
\Delta^{k} a_{n}=\left(2-\frac{4 n}{3}\right) \sum_{i=0}^{k}(-1)^{k+i}\left(\begin{array}{l}
k \\
i
\end{array}\right) i-\frac{2}{3} \sum_{i=0}^{k}(-1)^{k+i}\left(\begin{array}{l}
k \\
i
\end{array}\right) i^{2},
$$

and thus $\Delta^{k} a_{n}=0$ for each $k \geq 3$. However, $\Delta^{2} a_{n}=-\frac{4}{3}$ for each $n \in \mathbb{N}$, i.e., this sequence is not $(\Delta, 2)$-convex, but it is $(\Delta, k)$-convex for each $k \geq 3$. It follows that $(\Delta, k)$-convex sequence need not be $(\Delta, k-1)$-convex, and vice versa. For instance, the sequence $\{1,1,2,2,3,3, \ldots\}$ is $(\Delta, 1)$-convex, but not $(\Delta, 2)$-convex. Also,

$$
a_{n+k}-a_{n+k-1}-a_{n+1}+a_{n}=\frac{4}{3}(1-k)<0
$$

for all $k \geq 2$. Therefore, this sequence is not $k$-convex for any $k \geq 2$.

Example 3.3. For sequence $\left(a_{n}\right)_{1}^{\infty}=\left(\frac{1}{n}\right)_{1}^{\infty}$ it is easy to verify that

$$
\Delta^{k} a_{n}=\frac{(-1)^{k} k !}{\prod_{i=0}^{k}(n+i)}
$$

for each $k \in \mathbb{N}_{0}$ and $n \in \mathbb{N}$. Thus, $\left(\frac{1}{n}\right)_{1}^{\infty}$ is $(\Delta, k)$-convex for each $k=2 m$, $m \in \mathbb{N}_{0}$, and $(\Delta, k)$-concave for each $k=2 m+1, m \in \mathbb{N}_{0}$. Also, it is easy to verify that $\left(\frac{1}{n}\right)_{1}^{\infty}$ is $k$-convex for each $k \geq 2$, and thus it fulfills the inequality (2).

Example 3.4. The Fibonacci sequence $\left(F_{n}\right)_{0}^{\infty}$ is also an example of a sequence which is both $k$-convex and $(\Delta, k)$-convex. Since $\Delta^{k} F_{n}=F_{n-k}$ for each $k \in \mathbb{N}_{0}$ and $n \geq k$, the Fibonacci sequence $\left(F_{n}\right)_{0}^{\infty}$ is $(\Delta, k)$-convex for each $k \in \mathbb{N}_{0}$ and $n \geq k$. We also have that

$$
\begin{aligned}
F_{n+1} \leq F_{n}+\frac{1}{k}\left(F_{n+k}-F_{n}\right) & \Leftrightarrow k F_{n-1} \leq F_{n+k}-F_{n}, \\
F_{n+k-1} \leq F_{n}+\frac{k-1}{k}\left(F_{n+k}-F_{n}\right) & \Leftrightarrow \quad F_{n+k-1}-F_{n} \leq(k-1) F_{n+k-2}
\end{aligned}
$$

for each $n \in \mathbb{N}$. Both these inequalities may be verified by induction w.r.t $k \geq 2$, which yields that the Fibonacci sequence $\left(F_{n}\right)_{0}^{\infty}$ is $k$-convex for each $k \geq 2$. 


\section{Acknowledgments}

This note is a part of author's bachelor thesis written at the Institute of Mathematics, Faculty of Science, P. J. Šafárik University in Košice.

\section{References}

[1] H. Bor, A study on local properties of Fourier series, Nonlinear Anal. 57 (2004), 191-197.

[2] S. Wu, L. Debnath, Inequalities for convex sequences and their applications, Comput. Math. Appl. 54 (2007), 525-534.

[3] M. Duchoň, B. Riečan, Generalized moment problem in vector lattices, Novi Sad J. Math. 26 (1996), 53-61.

[4] J. Haluška, O. Hutník, On generalized weighted quasi-arithmetic means in integral form, Jour. Electrical Engineering 56 12/s (2005), 3-6.

[5] O. Hutník, On Hadamard's type inequalities for generalized weighted quasi-arithmetic means, J. Inequal. Pure Appl. Math. 7, No. 3 Art. 96 (2006).

[6] O. Hutník, Some integral inequalities of Hölder and Minkowski type, Colloq. Math. 108, No. 2 (2007), 247-261.

[7] J. Haluška, O. Hutník, Some inequalities involving integral means, Tatra Mt. Math. Publ. 35 (2007), 131-146.

[8] L. M. Kocić, On generalized convexity preserving matrix transformation, Univ. Beograd. Publ. Elektrotehn. Fak. Ser. Mat. Fiz. 762 (1982), 3-10.

[9] I. B. Lacković, M. R. Jovanović, On a class of real sequences which satisfy a difference inequality, Univ. Beograd. Publ. Elektrotehn. Fak. Ser. Mat. Fiz. 678 (1980), 99-104.

[10] A. Lupaş, On the means of convex sequences, Gazeta Matematiča, Seria (A) IV, No-s: 1, 2 (1983), 90-93.

[11] A. McD. Mercer, Polynomials and convex sequence inequalities, J. Inequal. Pure Appl. Math. 6, No. 1 Art. 8 (2005). 
[12] C. R. Selvaraj, Convexity preserving summability matrices, Internat. J. Math. Math. Sci. 13, No. 3 (1990), 501-506.

[13] Gh. Toader, On some properties of convex sequences, Math. Vesnik 38 (1986), 103-111.

[14] Gh. Toader, On the convexity of high order of sequences, Publications de L'Institut Mathématique 43, No. 57 (1988), 35-40.

[15] L.-Ch. Wang, Monotonicity and convexity of four sequences originating from Nanson's inequality, J. Inequal. Pure Appl. Math. 7, No. 4, Art. 150 (2006). 